\title{
Acute Refractory Hypotension 24 Hours Post-Inferior Vena Cave Filter Insertion Complicated by Early Phlegmasia Cerulea Dolens
}

\author{
Dou-Anne Siew ${ }^{\mathrm{a}}$, Daniele Wiseman ${ }^{\mathrm{b}}$, Richard Hilsden ${ }^{\mathrm{a}}$, Ranko Bulatovic ${ }^{\mathrm{a}}$, \\ Raymond $\mathrm{Kao}^{\mathrm{a}, \mathrm{c}}$
}

\begin{abstract}
We present a case of a 67-year-old man with T4aN3bM0 gastric adenocarcinoma who developed refractory hypotension 24 hours postinferior vena cave filter (IVCF) insertion for a new finding of pulmonary embolism (PE) and ongoing melena stool. After 18 hours of aggressive $20 \mathrm{~L}$ intravenous fluid resuscitation in the ICU followed by vasopressors and intravenous antibiotics, a point-of-care echocardiography did not reveal right ventricular strain and a non-contrast CT scan of the abdominal and pelvis was reported as not remarkable. But his lower limbs became progressively edematous and swollen while his upper limb maintained its normal circumference. At 24 hours post-ICU admission, his lower limbs had worsened with severe pain and pallor. The compartmental pressure measurements were in the high normal ranges. At morning rounds, the complete occlusion of the IVCF causing severe hypovolemic shock was considered and re-review of the non-contrast CT abdomen and pelvis showed complete collapsed IVC above the IVCF. The IVC below the IVCF was distended, as were the common and external iliac veins bilaterally concerning for extensive venous thrombosis and early phlegmasia cerulea dolens (PCD). The patient was treated with local catheterdirected thrombolysis with thrombus maceration and aspiration followed by localized direct tissue plasminogen activator (tPA) infusion and systemic heparin infusion. The patient's refractory hypotension resolved quickly and had surgery to remove his gastric tumor 8 days post-IVCF insertion. The placement of IVCF in a patient with known malignancy can greatly increase the risk for additional thrombus formation. But in our patient the speed is unprecedented, in which complete vena cava thrombosis (VCT) occurred at the site of the IVCF and below and its mechanism of causing refractory hypotension is rare. The consideration of PCD in a patient with refractory hypovolemic shock post-IVCF insertion followed by aggressive fluid resus-
\end{abstract}

Manuscript accepted for publication June 30, 2015

aLondon Health Sciences Center, Division of Critical Care and Department of Medicine, University of Western Ontario, London, Ontario, Canada

bLondon Health Sciences Center, Department of Radiology, University of Western Ontario, London, Ontario, Canada

${ }^{\mathrm{c} C o r r e s p o n d i n g ~ A u t h o r: ~ R a y m o n d ~ K a o, ~ L o n d o n ~ H e a l t h ~ S c i e n c e s ~ C e n t e r, ~ D i-~}$ vision of Critical Care and Department of Medicine, University of Western Ontario, 800 Commissioner's Rd E, London, Ontario N6A 5W9, Canada.

Email: Raymond.Kao@lhsc.on.ca

doi: http://dx.doi.org/10.14740/jmc2204w citation and worsening lower limb pain and discoloration remains a rare but important differential diagnosis.

Keywords: Inferior vena cava filter; Vena cava thrombosis; Pulmonary embolism; Refractory hypotension; Phlegmasia cerulea dolens; Catheter-directed thrombolysis

\section{Introduction}

The placement of inferior vena cava filter (IVCF) in a patient with known malignancy can greatly increase the risk for additional thrombus formation. But in our patient the speed is unprecedented in which complete vena cava thrombosis (VCT) occurred within $24 \mathrm{~h}$ of insertion at the site of the IVCF and below. This complete VCT is a rare cause of acute refractory hypotension and a delay in its diagnosis resulted in high volume fluid resuscitation, high dose vasopressors utilization and early complication of phlegmasia cerulea dolens (PCD).

\section{Case Report}

A 67-year-old man presented with a newly diagnosed stage T4aN3bM0 gastric adenocarcinoma. His past medical history included type 2 diabetes, hypertension, hyperlipidemia, gastroesophageal reflux disease, and coronary artery disease with bypass surgery 3 years prior to this admission. He was employed as a park manager and an ex-smoker with a 35-pack-year smoking history. He denied alcohol or illicit drug use. There was no significant family history of medical illnesses. While awaiting elective curative intent surgery, he developed gastric outlet obstruction and melena stools. He was transferred to our tertiary center for an urgent subtotal gastrectomy. A repeat staging CT scan for the abdomen and chest 30 days after his initial CT scan revealed new findings of bilateral pulmonary embolism (PE). The gastric outlet obstruction and the presence of extensive regional lymphadenopathy were also confirmed.

In preparation for his upcoming surgery and presence of new PE, an IVCF was inserted pre-operatively by interventional radiology via a right internal jugular vein access. Due to ongoing melena stools, heparin was not prescribed postIVCF insertion. Bilateral lower limb ultrasound was negative 


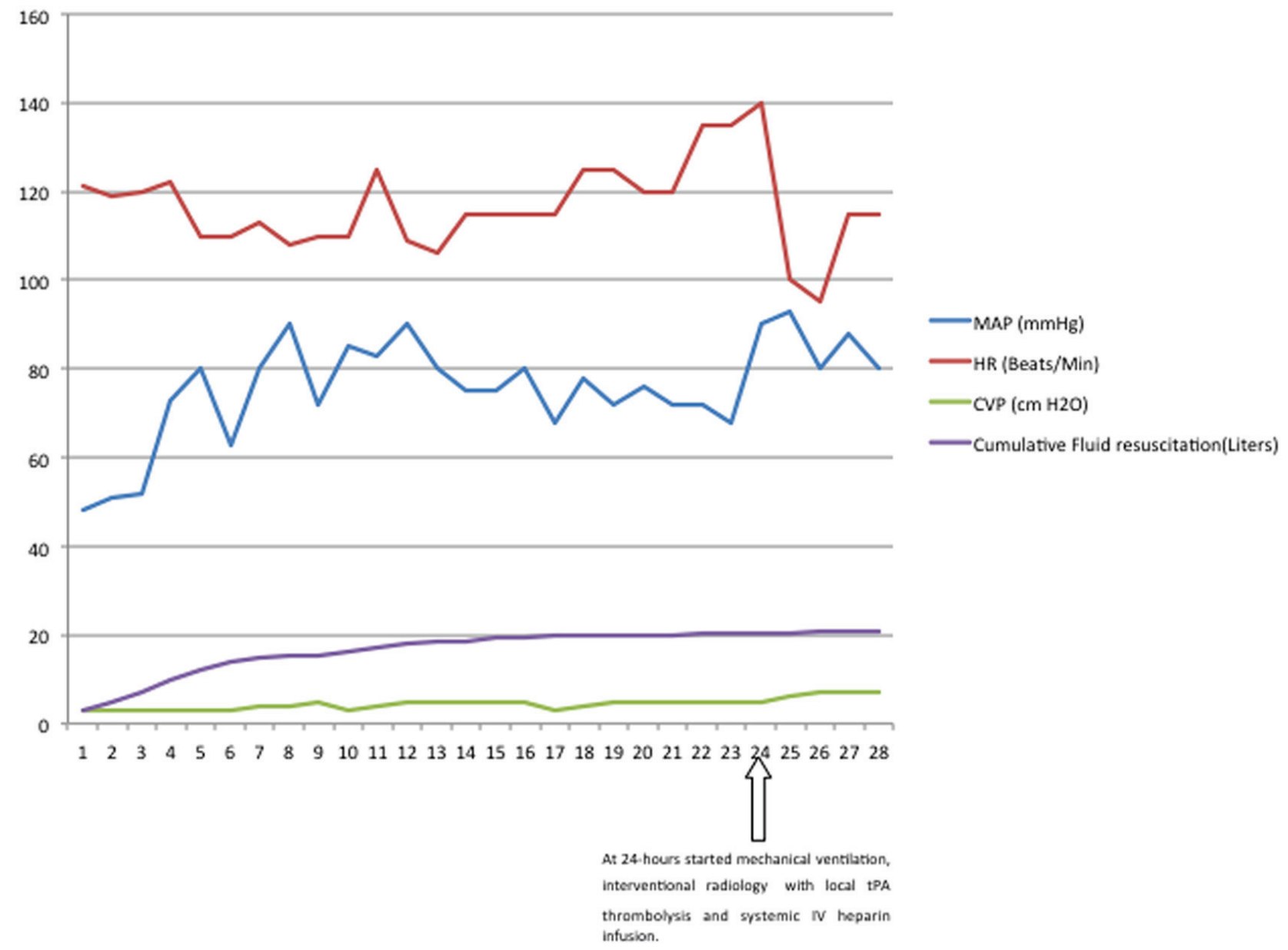

Figure 1. Twenty-eight hours hemodynamic monitoring for mean arterial pressure (MAP, $\mathrm{mm} \mathrm{Hg}$ ), heart rate (HR, beats/min), central venous pressure (CVP, $\mathrm{cm} \mathrm{H} 2 \mathrm{O}$ ) and cumulative fluid resuscitation (L).

for deep vein thrombosis (DVT). A peripherally inserted central catheter was inserted 1 day prior to the IVCF placement and parenteral nutrition was subsequently started. Twenty-four hours after IVCF placement, the patient developed sudden-onset hypotension and diaphoresis, his heart rate was 125 beats/ min, blood pressure was $60 / 40 \mathrm{~mm} \mathrm{Hg}$, and his oxygen saturation was unreadable. He had good air entry bilaterally, a soft non-tender abdomen, and denied chest pain or shortness of breath. The patient was given $1 \mathrm{~L}$ bolus of lactate ringer's solution and transferred urgently to the ICU. The patient continued to be hypotensive in the ICU and required large volume fluid resuscitation supplemented with vasopressors, norepinephrine and vasopressin (Fig. 1). A point-of-care echocardiogram performed in the ICU demonstrated a hyperdynamic left ventricle with no sign of right ventricular strain to suggest worsening PE. After initial fluid resuscitation with $8 \mathrm{~L}$ of crystalloid, the patient had a measured central venous pressure (CVP) of $3 \mathrm{~cm}$ $\mathrm{H}_{2} \mathrm{O}$. After instituting vasopressor therapy, his CVP remained low at $5 \mathrm{~cm} \mathrm{H}_{2} \mathrm{O}$, with a stroke volume variation (SVV) between $33 \%$ and $55 \%$ consistent with ongoing hypovolemia.

During his first $18 \mathrm{~h}$ in the ICU, he received a total of 20 L of crystalloid; however, he remained refractory to weaning from norepinephrine (maximum $15 \mu \mathrm{g} / \mathrm{min}$ ), vasopressin $(2.4$ $\mathrm{U} / \mathrm{h})$ and phenylephrine $(200 \mu \mathrm{g} / \mathrm{min})$. During these events, the patient remained chest pain free, alert and oriented, denying dyspnea, abdominal pain or confusion. His oxygenation was maintained on $2 \mathrm{~L}$ by nasal prong, and his heart rate was between 110 and 120 beats/min. His only complaint was that his legs were increasingly painful and swollen. He had no evidence of bleeding or worsening hematemesis. Nasogastric output was $4 \mathrm{~L}$ over the course of $24 \mathrm{~h}$.

The patient was pan cultured, and given intravenous stress-dose steroids and broad-spectrum antibiotics for possible septic shock. An urgent abdomen/pelvis CT scan without IV contrast was performed, and reported as unremarkable. Throughout the night as he received repeated fluid boluses, his lower limbs became cool, edematous, and exceedingly painful. His pulses remained palpable. The next morning due to his worsening lower limb tenderness and pallor, plastic surgery was consulted to rule out compartment syndrome. His compartment pressures were 15 and $17 \mathrm{~cm} \mathrm{H}_{2} \mathrm{O}$ at the high end of normal. During morning rounds, the possibility of IVCF occlusion causing refractory hypotension was brought up. On re-examination of the abdomen/pelvis CT scan by the radiol- 

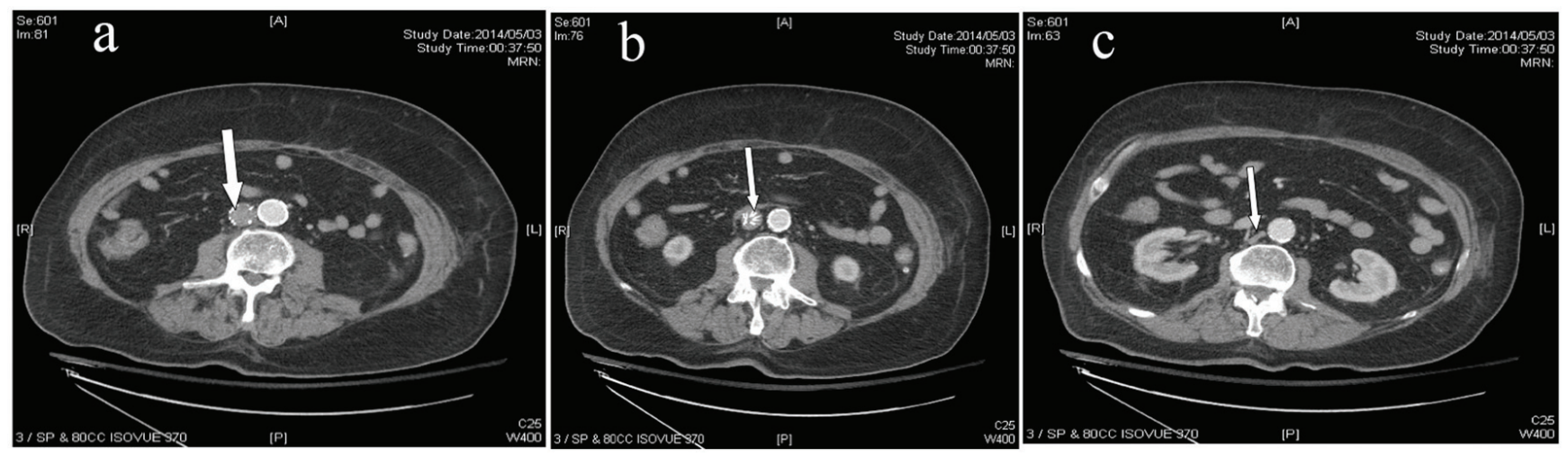

Figure 2. (a) Patient IVC below IVC filter (arrow). (b) IVC filter (arrow). (c) Complete collapse superior to the IVC filter (arrow).

ogy consultant, a completely collapsed IVC above the IVCF was seen. The IVC below the IVCF was distended, as were the common and external iliac veins bilaterally concerning for extensive venous thrombosis (Fig. 2a, b, c). An urgent lower limb Doppler ultrasound confirmed extensive bilateral lower limb DVT. The patient was taken emergently to interventional radiology for catheter-directed thrombolysis with tissue plasminogen activator (tPA). A bilateral common femoral venogram confirmed extensive thrombus throughout both femoral, iliac veins and the entire IVC below the filter. The patient received catheter-directed tPA pulse sprayed via long infusion catheters placed bilaterally and underwent maceration of clot by spinning a 5 French pigtail catheter. An overnight localized tPA infusion was started via the $30 \mathrm{~cm}$ right and $20 \mathrm{~cm}$ left infusion catheters with overlap in the IVC. A modified systemic heparin infusion was also instituted aiming for an aPTT of 50 $70 \mathrm{~s}$ consistent with local institution thrombolysis procedures. The tPA infusion was initially set at a total $2 \mathrm{mg} / \mathrm{h}$ which was reduced after $2 \mathrm{~h}$ instead at $6 \mathrm{~h}$ to $1 \mathrm{mg} / \mathrm{h}$ due to the onset of melena and hematuria from a traumatic Foley catheter insertion. After overnight tPA infusion, further pulse spray with tPA, mechanical maceration of clot with pigtail catheter and mechanical suction of clot was performed via long bilateral 12 French sheaths.

After the start of tPA therapy, vasopressors were immediately weaned off. Due to blood loss during thrombolysis, the patient required transfusion of 4 Units PRBC. Twenty-four hours after initiation of thrombolysis, a recheck by interventional radiology revealed resolution of the right sided iliac and femoral clot, but ongoing left-sided common iliac vein occlusion that was likely more chronic, based on collaterals (Fig. $3 a, b, c)$. The IVC continued to show a large non-occlusive thrombus in the filter itself but the remainder had cleared. The patient was continued on therapeutic intravenous heparin without further localized tPA and subsequently received surgical resection of his gastric cancer 8 days post-IVCF thrombus formation. He was transferred to the general surgery floor from the ICU with no recurrence of hemodynamic compromise during the remainder of his admission.

\section{Discussion}

Our patient presented with refractory hypotension $24 \mathrm{~h}$ postIVCF placement. The patient's SVV suggested hypovolemia and his hemodynamic indices would temporarily respond to fluid resuscitation; however; despite receiving more than 20 $\mathrm{L}$ of crystalloid, the improvement in hemodynamics was only transient. The patient was treated for possible severe sepsis, and other causes for his refractory hypotension were entertained. We also considered a diagnosis of vasoplegia, but the patient did not have any history suggesting treatment with antihypertensive medications, drug overdose, electrolyte disturbances such as severe hypermagnesemia, anaphylaxis, severe

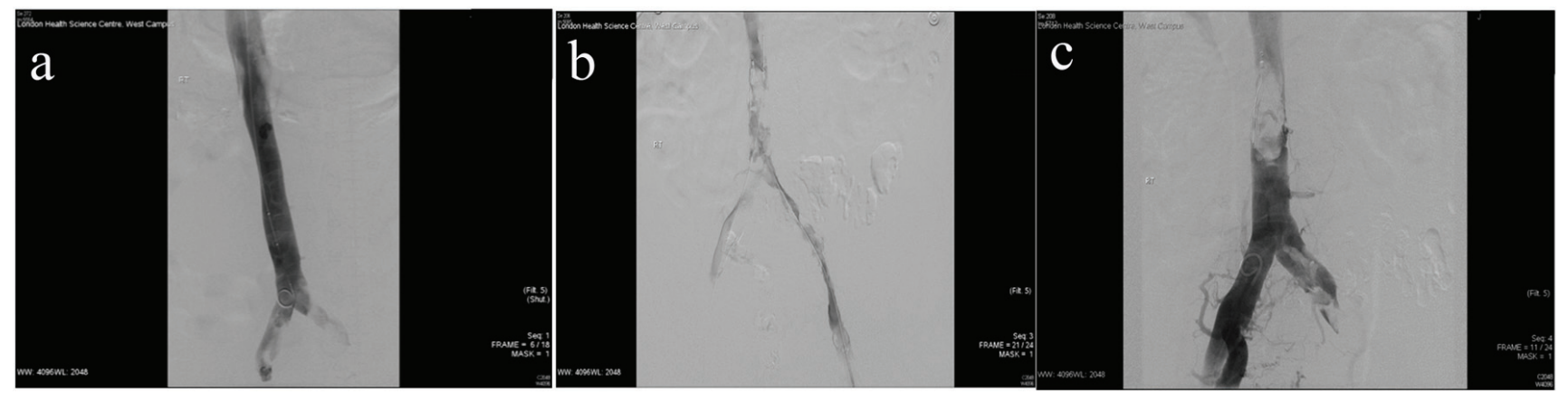

Figure 3. (a) Post-IVC filter insertion, with no evidence of thrombus. (b) Extensive clot burden in IVC filter, femoral and iliac veins during thrombolysis. (c) Twenty-four hours post-thrombolysis revealed ongoing left femoral occlusion and non-occlusive IVC filter thrombus. 
systolic dysfunction with LVEF $<35 \%$, or chronic liver disease $[1,2]$. The CT scan showed evidence of significant IVC thrombus. Thus, given his bedside echo that did not support a diagnosis of massive $\mathrm{PE}$, it became clear that his hypotension was due to reduced venous return resulting in markedly inadequate preload. The fact that such rapid and extensive thrombus formation occurred after IVCF insertion is unusual.

IVCFs are used to prevent PE in the presence or absence of venous thromboembolic events (VTEs) [3]. Often, IVCFs are used when there is contraindication to systemic anticoagulation and an ongoing risk for PE [3]. In the short term, the benefit of preventing life-threatening PE is thought to outweigh the risk of new thrombus generation. One single center review found that the prevalence of VCT occurred in only $2.7 \%$ of patients [4]. But a retrospective study examining the incidence of VCT after IVCF placement reported a $22 \%$ incidence over a median follow-up of 24 months, compared to $7 \%$ in the group who did not receive IVCF [5]. The average time between IVCF placement and VCT was 6.4 months (range 2 - 26 months) [5]. In most instances, VCT occurred after cessation of anticoagulation treatment and most were asymptomatic; however, two VCT-related fatalities presented with PCD and abdominal compartment syndrome [5]. Also IVCF has been subject to one randomized controlled trial evaluating thrombosis rate reduction. A statistically significant reduction of $\mathrm{PE}$ at the 12day mark was observed with IVCF insertion compared with anticoagulation alone; however, this effect was lost upon follow-up at 2 years [6]. Furthermore, all patients in the study received anticoagulation, and in addition for those who received an IVCF had a significantly higher risk of recurrent lower limb DVT at 2-year follow-up, with no difference in 2-year mortality rate [6]. It is known IVCFs are thrombogenic. One review reported IVC thrombosis rates of 3.6-11.2\% and insertion-site thrombosis of $23-36 \%$ [7]. In our patient, extensive thrombi formation from the IVCF to both lower extremities occurred after IVCF placement, as initial bilateral leg Doppler ultrasound on admission was negative. Our patient also had newly diagnosed, untreated gastric cancer. Malignancy is associated with an increased risk of VTE. A prospective database examining gastric cancer patients in a Korean center found a 2-year cumulative incidence of VTE after diagnosis of 3.8\% in stage III patients [8]. The 2-year incidence of DVT/PE was $0.8 \%$ for stage II-IV (M0) patients. DVT/PE was a significant predictor of earlier death in these patients $[8,9]$.

Our patient developed refractory hypovolemic shock within $24 \mathrm{~h}$ of IVCF insertion, which required large volume fluid resuscitation, which may have resulted in early PCD. PCD was first described in 1593 when DVT would result in venous gangrene [10]. Historically it has a reported $20-40 \%$ mortality rate [10]. Nearly 300 years later it is recognized as resulting from massive occlusion of large and small caliber veins [10]. It is a rare complication of DVT thought to occur from a dysregulation between coagulation and fibrinolysis. Clinically, patients experience the acute onset of cyanosis starting distally, significant pain, edema and bullae, eventually resulting in loss of arterial pulses and sensation, paralysis, and venous gangrene, necessitating amputation [10]. We suggest that in our patient, given his expeditious localized thrombolysis, thrombus maceration and aspiration, the severe symptoms and fatality of PCD did not occur.

The exact mechanisms and pathophysiology of PCD are still unclear. The most often quoted paper from 1967 examined the hemodynamics involved in massive peripheral venous occlusion in six anesthetized canines. This study found that within $6 \mathrm{~h}$ of sudden and extensive femoral and iliac vein occlusion, venous hypertension of the involved limb occurred. This led to elevated tissue pressures and extravasation of fluid from the venous system into the surrounding tissue due to disruption of osmotic and hydrostatic pressures. The ongoing fluid accumulation into the interstitial space increased tissue pressures and limb volume, and led to significant hypovolemia which briefly responded to fluid resuscitation. After $24 \mathrm{~h}$ of extensive venous occlusion, the hydrostatic pressure in two animals was $40 \mathrm{~mm}$ $\mathrm{Hg}$ and $52 \mathrm{~mm} \mathrm{Hg}$. Iliac flow was lost at that point and absent arterial pulse was also noted [12]. It is postulated that initially venous collaterals are able to maintain adequate arterial tissue perfusion thus preventing tissue ischemia and gangrene. Progressively, arterial flow becomes more compromised, leading to loss of peripheral pulses, then peripheral limb ischemia and gangrene. Case reports have shown that patients can accumulate up to $6-10 \mathrm{~L}$ of fluid within their lower limb tissue within 5 - 10 days [13]. Our patient's worsening lower leg pain, pallor and edema suggest that venous hypertension and extravasation of fluid from his lower venous system occur, resulting in profoundly diminished preload and his seemingly refractory hypovolemic shock. This hypothesis is also supported by the immediate improvement in his hemodynamics once thrombolysis, clot maceration and removal occurred.

The treatment of extensive VCT and/or PCD includes surgical thrombectomy, percutaneous manual aspiration thrombectomy [14], catheter-directed and systemic thrombolysis, which is most widely used [16]. The first case using thrombolytics in the treatment of PCD was published in 1970 [15]. More recently, thrombolytic therapy has been suggested to be curative of PCD compared to surgical thrombectomy. A prospective multicenter trial evaluated catheter-directed thrombolysis and found higher rates of clot lysis achieved with locally directed urokinase or tPA than anticoagulation alone [16]. However, high rates of major bleeding (11\%) prevent this option from being used widely [16].

\section{Conclusion}

The placement of IVCF in a patient with known malignancy may greatly increase the risk for additional thrombus formation. But in our patient the speed is unprecedented in which complete VCT occurred at the site of the IVCF and below and its mechanism of causing refractory hypotension is rare. The consideration of PCD in a patient with refractory hypovolemic shock post-IVCF insertion with aggressive fluid resuscitation and worsening lower limb pain and discoloration remains a rare but important differential diagnosis.

\section{References}

1. Levin MA, Lin HM, Castillo JG, Adams DH, Reich DL, 
Fischer GW. Early on-cardiopulmonary bypass hypotension and other factors associated with vasoplegic syndrome. Circulation. 2009;120(17):1664-1671.

2. Mordes JP, Swartz R, Arky RA. Extreme hypermagnesemia as a cause of refractory hypotension. Ann Intern Med. 1975;83(5):657-658.

3. Kinney TB. Inferior vena cava filters. Semin Intervent Radiol. 2006;23(3):230-239.

4. Athanasoulis CA, Kaufman JA, Halpern EF, Waltman AC, Geller SC, Fan CM. Inferior vena caval filters: review of a 26-year single-center clinical experience. Radiology. 2000;216(1):54-66.

5. Guo YJ, Feng J, Qu TR, Qu Y, Liu YM, Zhang YS, Tian HY, et al. Vena cava thrombosis after vena cava filter placement: Incidence and risk factors. J Geriatr Cardiol. 2011;8(2):99-103.

6. Decousus H, Leizorovicz A, Parent F, Page Y, Tardy B, Girard P, Laporte S, et al. A clinical trial of vena caval filters in the prevention of pulmonary embolism in patients with proximal deep-vein thrombosis. Prevention du Risque d'Embolie Pulmonaire par Interruption Cave Study Group. N Engl J Med. 1998;338(7):409-415.

7. Streiff MB. Vena caval filters: a comprehensive review. Blood. 2000;95(12):3669-3677.

8. Lee KW, Bang SM, Kim S, Lee HJ, Shin DY, Koh Y, Lee $\mathrm{YG}$, et al. The incidence, risk factors and prognostic implications of venous thromboembolism in patients with gastric cancer. J Thromb Haemost. 2010;8(3):540-547.

9. Tetzlaff ED, Correa AM, Baker J, Ensor J, Ajani JA. The impact on survival of thromboembolic phenomena occur- ring before and during protocol chemotherapy in patients with advanced gastroesophageal adenocarcinoma. Cancer. 2007;109(10):1989-1995.

10. Stallworth JM, Bradham GB, Kletke RR, Price RG, Jr. Phlegmasia Cerulea Dolens: A 10-Year Review. Ann Surg. 1965;161:802-811.

11. Suwanabol PA, Tefera G, Schwarze ML. Syndromes associated with the deep veins: phlegmasia cerulea dolens, May-Thurner syndrome, and nutcracker syndrome. Perspect Vasc Surg Endovasc Ther. 2010;22(4):223-230.

12. Snyder MA, Adams JT, Schwartz SI. Hemodynamics of phlegmasia cerulea dolens. Surg Gynecol Obstet. $1967 ; 125: 342-346$.

13. Bedri MI, Khosravi AH, Lifchez SD. Upper extremity compartment syndrome in the setting of deep venous thrombosis and phlegmasia cerulea dolens: case report. J Hand Surg Am. 2009;34(10):1859-1863.

14. Oguzkurt L, Ozkan U, Demirturk OS, Gur S. Endovascular treatment of phlegmasia cerulea dolens with impending venous gangrene: manual aspiration thrombectomy as the first-line thrombus removal method. Cardiovasc Intervent Radiol. 2011;34(6):1214-1221.

15. Paquet KJ, Popov S, Egli H. [Guidelines and results of consequent fibrinolytic therapy in phlegmasia cerulea dolens]. Dtsch Med Wochenschr. 1970;95(16):903-904.

16. Mewissen MW, Seabrook GR, Meissner MH, Cynamon J, Labropoulos N, Haughton SH. Catheter-directed thrombolysis for lower extremity deep venous thrombosis: report of a national multicenter registry. Radiology. 1999;211(1):39-49. 